\title{
Importance of intermediate water formation for suplying iron and macro-nutrient in the North Pacific
}

\author{
JUN NiSHIOKA ${ }^{1,}$ HAJIME OBATA ${ }^{2}$, ICHRO YASUDA ${ }^{2}$
}

${ }^{1}$ Institute of low temperature science, Hokkaido University

${ }^{2}$ Atmosphere and Ocean Research Instietute, The University of Tokyo

The subarctic North Pacific is high nutrient low chlorophyll region, where phytoplankton growth is limited by iron (Fe) availability, and the area has high bio- $\mathrm{CO}_{2}$ drawdown. The overall picture of the processes for supplying $\mathrm{Fe}$ and macro-nutrients, however, are still not fully understood. We compiled comprehensive observed data in the North Pacific in this 20 years, including the marginal seas and its island chains (IC), and construct a dissolved Fe (DFe) dataset with nutrients and hydro-cast water properties. The dataset can withstand the analysis of the distribution of the chemical parameter on isopycnal surface. Then, we constructed 3D DFe diagram of the North Pacific including subpolar marginal seas by using the dataset. The isopycnal analysis indicates that the DFe rich water propagate from the Okhotsk Sea Intermediate Water to the wide area of Upper(U)-NPIW (26.6-27.0 $\sigma \theta$ ) in the western North Pacific, especially from $155 \mathrm{E}$ to the west. Extremely nutrient-rich water is pooled in intermediate water $(26.8-27.6 \sigma \theta)$ in the subarctic area, especially in the Bering Sea basin. Increases of two to four orders in the upward turbulent fluxes of nutrients were observed around the marginal sea island chains, indicating that nutrients are uplifted to the surface and are returned to the subarctic intermediate nutrient pool (SINP) as sinking particles through the biological production and microbial degradation of organic substances. The nutrient circulation in the SINP coupled with the DFe in Uintermediate water derived from the Okhotsk Sea (external $\mathrm{Fe}$ input) leads to a relatively high $\mathrm{DFe}: \mathrm{N}$ ratio in the intermediate waters. The $\mathrm{DFe}: \mathrm{N}$ ratios are higher in the Okhotsk Sea and around the Kuril ICs in the subsurface to intermediate density ranges than that around the Aleutian ICs and in the Bering Sea basin, indicating that the Fe-rich water diapycnally upwells to the surface around the Kuril ICs by strong turbulent mixing. Our results clearly indicate that, in the subarctic Pacific, sub-polar marginal seas and intermediate waters play pivotal roles for surface biogeochemistry and are involved in formation of the area which has the largest bio- $\mathrm{CO}_{2}$ drawdown in the world ocean. 